\title{
Early adolescent Depo-Provera exposure increases stillbirths in adult sooty mangabeys
}

\author{
Maurand Cappelletti, Kelly Ethun ${ }^{1}$, Tracy Meeker ${ }^{1}$, Gretchen Von Scherr ${ }^{1}$ and Kim Wallen \\ Department of Psychology, Emory University, 36 Eagle Row, Atlanta, Georgia 30322, USA and ${ }^{1}$ Yerkes National \\ Primate Research Center, Atlanta, Georgia 30322, USA \\ Correspondence should be addressed to M Cappelletti; Email: m.m.cappelletti@emory.edu
}

\begin{abstract}
The 3-month injectable contraceptive medroxyprogesterone acetate (MPA; Depo-Provera) is a synthetic progestin that protects against pregnancy by suppressing ovulation. Studies have focused on the resumption of ovulation after MPA-treatment cessation but neglected potential long-term effects of MPA exposure on future successful reproduction. MPA is frequently administered to adolescent girls; however, long-term fertility effects of adolescent MPA exposure have not been explored. We investigated fertility after extended MPA exposure in a species of old world primate, the sooty mangabey (Cercocebus atys). Female sooty mangabeys $(n=31)$ received chronic MPA-treatment for 4-8 years. At MPA-treatment onset, subjects were either parous adults $(n=14)$ or nulliparous adolescents $(n=17)$, with adolescent-treated subjects being further divided into those who had reached first ovulation $(n=10)$ and those who had not $(n=7)$. After MPA-treatment cessation, adolescent-treated females had a significantly higher incidence of stillbirth than did age-matched and parity-matched controls, whereas adult-treated females did not differ from their matched controls. Females placed on MPA-treatment prior to first ovulation had a significantly higher incidence of stillbirth post-treatment than did females placed on MPA-treatment after first ovulation. Diabetic females had an increased incidence of stillbirth as compared to nondiabetic females; however, when controlling for diabetes, MPA exposure prior to first ovulation was still a significant positive predictor of stillbirth. These findings suggest that the post-treatment fertility effects of chronic MPA exposure vary with the developmental timing of treatment onset and raise concern about the use of MPA as a contraceptive for adolescent girls.

Reproduction (2015) 150 497-505
\end{abstract}

\section{Introduction}

The 3-month injectable contraceptive medroxyprogesterone acetate (MPA; Depo-Provera) is a synthetic progestin that protects against pregnancy by acting at the level of the pituitary and hypothalamus to suppress ovulation (Rivera et al. 1999). MPA is currently approved for human use in over 90 countries worldwide (Khoiny 1996), and since its approval by the Food and Drug Administration (FDA) in 1992, over 11 million women in the United States have used MPA (Mosher \& Jones 2010).

Return of fertility after MPA-treatment cessation is of concern to both MPA users and prescribing physicians; however, studies examining fertility after MPA exposure have largely focused on resumption of ovulation. Some evidence suggests that women previously exposed to MPA may be at an increased risk of adverse pregnancy outcomes. In a single cohort of women living on the island of Jamaica, Greenwood and McCaw-Binns (1994) and Greenwood et al. (1994) reported that previous Depo-Provera use (modeled with maternal age) was a significant positive predictor of stillbirth, even when controlling for factors such as drug and alcohol use and maternal nutrition; however, such associations can be difficult to interpret in women given an unavoidable lack of environmental controls.

Many clinicians recommend prescribing MPA instead of oral contraceptives specifically for adolescent patients to avoid compliance issues associated with a daily pill regimen (Cromer et al. 1998, Khoiny 1996, Tolaymat \& Kaunitz 2007). Between 2006 and 2008, over 270000 adolescent girls (ages 15-19) in the United States alone were using MPA (Mosher \& Jones 2010). Despite the large number of adolescent MPA users, the possibility of differential long-term fertility effects of MPA exposure during adolescence has not been explored.

Captive nonhuman primates provide an opportunity to investigate the long-term fertility effects of synthetic progestin exposure in a controlled environment. No studies have explicitly investigated the effects of MPA exposure on future infant survival in nonhuman primates; however, two studies examined the fertility effects of exposure to a similar synthetic progestin, melengestrol acetate (MGA). Unlike MPA, MGA is administered via subdermal implant; however, both 
contraceptives protect against pregnancy via the same mechanism. Wood et al. (2001) reported that female golden lion tamarins (Leontopithecus rosalia) exposed to MGA had a significantly higher risk of stillbirth after MGA-treatment cessation than did nonexposed controls. A portion of their sample, however, was comprised of females whose MGA capsules had been exhausted but not surgically removed. These "empty" MGA capsules could have been releasing low levels of MGA during pregnancy, making the results of this study difficult to interpret. De Vleeschouwer et al. (2000) examined fertility after MGA exposure in golden-headed lion tamarins (Leontopithecus chrysomelas) but limited their analysis to females whose MGA capsules had been surgically removed. The authors reported that females had a significantly higher risk of stillbirth after MGAtreatment cessation as compared to before they were exposed to MGA; however, these females were also several years older after MGA treatment as compared to before, and the authors did not control for maternal age. Nonetheless, both of these studies suggest the need for further investigation into the long-term effects of synthetic progestin exposure on future infant survival in nonhuman primates.

The present study examined the return of fertility, reproductive output, and pregnancy outcomes after extended MPA exposure in a species of the old-world primate, the sooty mangabey (Cercocebus atys). Sooty mangabeys are menstrual primates with an average menstrual cycle length within the normal human range (Hadidian \& Bernstein 1979) and patterns of hormonal fluctuation across the menstrual cycle very similar to those in women (Aidara et al. 1981). Sooty mangabeys therefore represent an appropriate animal model for the study of processes relevant to human reproductive endocrinology. We report here that the fertility consequences of extended MPA exposure in sooty mangabeys varied with the developmental timing of MPA-treatment onset.

\section{Materials and methods}

Data in this study were obtained from historical clinical records of the Yerkes National Primate Research Center. All clinical procedures described in this study were approved by the Emory University Institutional Animal Care and Use Committee under the Maintenance of the Yerkes National Primate Center Animal Colony Protocol (\#2003042).

\section{Treated subjects}

MPA-treated subjects

Treated subjects were female sooty mangabeys (Cercocebus atys; $n=31$ ) living in one large multi-male/multifemale social group at the Yerkes National Primate
Research Center (YNPRC) Field Station in Lawrenceville, GA, USA, housed in a large outdoor enclosure $\left(\sim 230 \mathrm{~m}^{2}\right)$ with attached indoor quarters $\left(\sim 16.5 \mathrm{~m}^{2}\right)$. All treated subjects were placed on MPA-treatment for population management, and not for health concerns. At the time of MPA-treatment onset, treated subjects were either parous adults ( $n=14 ; 1-3$ births) or nulliparous adolescents $(n=17)$, with adolescent-treated subjects being further defined as ovulatory $(n=10)$ or preovulatory $(n=7)$. Following an established protocol for estimating ovulation in sooty mangabeys, the last day of maximal perineal swelling during a given menstrual cycle was considered the day of ovulation (Aidara et al. 1981). Adolescent-treated subjects were defined as preovulatory if they had never achieved maximal perineal swelling when MPA-treatment began. Menstruation data were not available, and the pre-ovulatory adolescenttreated subjects may or may not have reached menarche at the time of MPA-treatment onset. The reproduction of each treated subject was examined via clinical records after MPA-treatment cessation $(M=1.97 \pm 0.64$ years $)$.

\section{MPA-treatment administration}

Treated subjects received original brand Depo-Prover and/or generic depot medroxyprogesterone acetate (obtained from various suppliers) via i.m. injection every 1-3 months for durations lasting 4-8 years. Adult-treated subjects were, on average, older at the time of their first MPA injection, on MPA-treatment longer, and received a greater total amount of MPA over the course of the treatment period than did adolescenttreated subjects (Table 1).

MPA injection dosage varied between 15 and $300 \mathrm{mg}$ and increased on a calendar method that was not based on weight throughout the treatment period in an attempt to achieve a dosage that would continuously suppress ovulation in all treated females. Four adult-treated subjects became pregnant during the treatment period. One of these females unintentionally received a MPA injection while pregnant, and that pregnancy subsequently ended in a stillbirth. The other three females received no further MPA injections during gestation and/or lactation, and MPA injections were resumed after their infants were weaned. One adolescent-treated subject included in this study was the product of one of these unintended pregnancies during the treatment period. While this subject was born of a mother exposed to MPA, it is unlikely that this female was exposed to MPA prenatally or while nursing, as her mother received no MPA injections during gestation and/or lactation.

\section{Controls}

All adult female sooty mangabeys in the YNPRC Field Station breeding colony were placed on MPA-treatment starting in October 2001; therefore, there were no 
Table 1 Comparison of measures between adult- and adolescent-treated subjects.

\begin{tabular}{|c|c|c|c|c|c|}
\hline \multirow[b]{2}{*}{ Treatment group } & \multirow[b]{2}{*}{$n$} & \multicolumn{2}{|c|}{ Age at } & \multicolumn{2}{|c|}{ Total } \\
\hline & & $\begin{array}{l}\text { First treatment } \\
\text { (year) }\end{array}$ & $\begin{array}{l}\text { Last treatment } \\
\text { (year) }\end{array}$ & $\begin{array}{c}\text { Time on treatment } \\
\text { (year) }\end{array}$ & $\begin{array}{l}\text { Drug exposure } \\
(\mathrm{mg})\end{array}$ \\
\hline Adult-treated & 14 & $6.72 \pm 0.35$ & $14.82 \pm 0.39$ & $8.10 \pm 0.06$ & $5090.36 \pm 148.31$ \\
\hline Adolescent-treated & 17 & $3.25 \pm 0.07^{\mathrm{a}}$ & $9.08 \pm 0.29^{\mathrm{a}}$ & $5.83 \pm 0.26^{\mathrm{a}}$ & $4503.53 \pm 172.77^{b}$ \\
\hline
\end{tabular}

Mean \pm S.E.M.

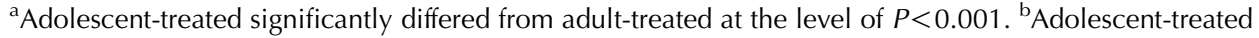

significantly differed from adult-treated at the level of $P<0.05$.

untreated females available to act as contemporary controls. Control females were randomly selected from the colony's historical records, and their reproduction was retrospectively examined during a time period prior to colony-wide MPA-treatment onset. As this was a retrospective observational study, the historical controls did not receive vehicle injections. No female acted as both a treated subject and a control.

\section{Age-matched controls}

To control for effects of maternal age on successful reproduction, an age-matched control was selected from historical records for each treated subject $(n=31)$. The reproduction of each age-matched control was retrospectively followed from the day when the control would have been the same age as their matched treated subject (e.g., if a treated subject was 3500 days old on the day of their first ovulation after MPA-treatment cessation, then the reproduction of their age-matched control was retrospectively followed from the historical day when the control would have also been 3500 days old). All age-matched controls had given birth at least once.

\section{Parity-matched controls}

The adolescent-treated subjects had all reached adulthood by the end of the MPA-treatment period but had still never given birth (unlike their parous age-matched controls). To control for effects of reproductive experience on successful reproduction, a parity-matched control was selected from historical records for each adolescent-treated subject $(n=17)$. The reproduction of each parity-matched control was retrospectively followed from the day of their first ovulation (i.e., all parity-matched controls were nulliparous adolescents).

\section{Following reproduction}

\section{Return of fertility}

Latency to return of fertility after MPA-treatment cessation was measured by both latency to resumption of ovulation (defined as the number of days from final MPA injection to first subsequent ovulation) and latency to first birth (defined as the number of days from final MPA injection to first subsequent birth).

\section{Reproductive output}

Reproductive output was defined as the total number of births across total number of days of reproductive opportunity during the study period. A given day was considered a day of reproductive opportunity if the subject spent the entire day in the group, with access to at least one fertile adult male. Any day that a subject spent out of the group was still considered a day of reproductive opportunity if the subject was already pregnant at the time. The reproductive output of each age-matched and parity-matched control was examined over the same number of days of reproductive opportunity as their matched treated subject.

\section{Pregnancy outcomes}

Each birth during the study period was categorized as one of the following pregnancy outcomes: live birth, planned C-section, stillbirth, neonatal death, maternal abuse/neglect, or premature birth (based on the definitions in Table 2). The percentage of total births during the study period categorized as each type of pregnancy outcome was calculated. All treated subjects had 0-3 births during the study period, and thus some treated subjects contributed more births toward the total count of births than did others. To account for the overrepresentation of certain treated subjects, each agematched and parity-matched control was followed for the same number of births as was their matched treated subject. To further account for the discrepancy in births contributed by each treated subject, all pregnancy outcome analyses were additionally performed for first births only during the study period.

\section{Perineal swelling data collection}

Trained observers visually inspected all female sooty mangabeys 5 days per week and rated perineal swelling size on a scale from 0 to 4 , with 4 indicating maximal swelling. Two observers collected all perineal swelling data used in this study with an inter-observer reliability of $85 \%$ agreement on swelling size. The same perineal 
Table 2 Definitions for categorization of pregnancy outcomes.

\begin{tabular}{|c|c|c|}
\hline Category & Type & Definition \\
\hline \multirow[t]{2}{*}{ Positive outcomes } & Live birth & Full-term infant delivered vaginally; survived more than 28 postnatal days \\
\hline & Planned C-section & $\begin{array}{l}\text { Full-term infant delivered via planned cesarean section; survived more than } \\
28 \text { postnatal days }\end{array}$ \\
\hline \multirow[t]{4}{*}{ Negative outcomes } & Stillbirth & Full-term infant born deceased (without having taken a breath) \\
\hline & Neonatal death & Infant died within 28 postnatal days for health reasons other than maternal abuse \\
\hline & Maternal abuse/neglect & $\begin{array}{l}\text { Full-term infant abused or neglected by dam; died or was removed from dam within } \\
28 \text { postnatal days }\end{array}$ \\
\hline & Premature birth & Pre-term infant; survived more than 28 postnatal days \\
\hline
\end{tabular}

swelling data collection protocol was used for all subjects, including historical controls.

\section{Diabetes}

Of the 31 treated subjects, 5 were determined to be diabetic during the study period. Diabetes has been shown to impact pregnancy outcomes in women (Lauenborg et al. 2003, Wood et al. 2003, Mathiesen et al. 2011), and we therefore examined the impact of diabetes on pregnancy outcomes in the treated subjects. Breeding female sooty mangabeys were determined to be diabetic if they met two of the four criteria: persistent hyperglycemia, elevated fructosamine, presence of glucosuria, and/or weight loss. Hyperglycemia was defined by a fasting blood glucose level greater than $175 \mathrm{mg} / \mathrm{dl}$ (Jones et al. 2014). Animals with fructosamine levels greater than $300 \mu \mathrm{mol} / \mathrm{l}$ were considered diabetic (Jones et al. 2014). The incidence of diabetes did not differ between the adult- $(14 \%)$ and adolescent-treated subjects $(18 \%$; Fisher's exact test (FET), degrees of freedom $(d f)=1, n=31, P=1.00, \quad \Phi=0.05)$. Diabetic mangabeys were not treated for their diabetes. Diabetes treatment requires daily monitoring of blood glucose levels and adjustments of medication dosage. These animals lived outside in large social groups, preventing daily accesses for blood collection. Furthermore, a major risk of treatment with diabetic drugs includes acute hypoglycemic episodes. In our experience, sooty mangabeys can live for years in a diabetic state without common diabetic sequelea, such as cardiovascular disease, nephropathy, and retinopathy. Thus, the risks of treatment for diabetes outweigh the benefits in these animals. Diabetes data were not available for historical controls.

\section{Statistical analysis}

All analyses used SPSS software (version 22.0.0, IBM Corp., Somers, NY, USA). All tests were two-tailed and considered significant with a $P$ value less than 0.05 . Group mean differences were assessed with the independent samples $t$-test, using Cohen's $d$ as a measure of effect size (Cohen 1977). Multiple linear regression was used to examine the relationship between parity at first treatment, total drug exposure, weight at last treatment, and latency to return of fertility after MPA-treatment cessation.
Differences between groups in the proportion of total births and first births with negative outcomes during the study period were analyzed with the Fisher's exact test (FET), using the phi coefficient as a measure of effect size (Cohen 1977). A binary logistic regression model (with pregnancies nested within dams) was used to examine the relationship between total drug exposure, ovulatory status at treatment onset, diabetes, and stillbirth. A oneway analysis of variance (ANOVA) was used to examine the relationship between birth number after final MPA injection (first, second, or third birth) and stillbirth. When attempting to balance the risk of type I versus type II error in our analyses, we decided that, given the exploratory nature of the present investigation and the fact that negative pregnancy outcomes are rare but highly significant events, the risk of type II error was more serious. We therefore maintained an alpha level of 0.05 but did not systematically correct for multiple comparisons (Bender \& Lange 2001).

\section{Results \\ Return of fertility}

One adult-treated female mangabey failed to resume ovulation within the study period after her final MPA injection (937 days) and was excluded from the study. Latency to resumption of ovulation after the final MPA injection varied between 106 and 317 days, and mean latency to resumption of ovulation did not differ between adult-treated ( $M=185.86$, s.D. $=55.80$ days $)$ and adolescent-treated subjects $(M=191.35$, S.D. $=45.34$ days, $t(29)=0.30, P=0.76$, Cohen's $d=0.11)$. Four adulttreated subjects failed to give birth again within the study period after their final MPA injections and were excluded from analysis of latency to first birth. Latency to first birth after the final MPA injection varied between 328 and 669 days, and mean latency to first birth also did not differ between adult-treated $(M=419.80$, s.D. $=$ 97.76 days) and adolescent-treated subjects $(M=$ 436.12, s.D. $=54.43$ days, $t(25)=0.56, P=0.58$, Cohen's $d=0.21$.

Parity at first treatment, total drug exposure, and weight at last treatment did not significantly predict latency to resumption of ovulation or latency to first birth after final MPA injection (Table 3). 
Table 3 Summary of multiple linear regression model predicting (a) latency to resumption of ovulation after final MPA injection and (b) latency to first birth after final MPA injection.

\begin{tabular}{lrrrc}
\hline Predictors & $\boldsymbol{B}$ & \multicolumn{1}{c}{ s.E.M. } & \multicolumn{1}{c}{$\boldsymbol{\beta}$} & $\boldsymbol{P}$ \\
\hline \multicolumn{4}{l}{ Latency to resumption of ovulation after final MPA } & injection \\
Constant & \\
Parity at first treatment & 217.33 & 97.98 & & 0.04 \\
Total drug exposure & 6.47 & 20.75 & 0.07 & 0.76 \\
Weight at last treatment & $<0.01$ & 0.02 & 0.02 & 0.93 \\
Latency to first birth after final MPA injection & -5.12 & 7.07 & -0.14 & 0.48 \\
Constant & 410.94 & 150.22 & & \\
Parity at first treatment & 27.22 & 33.06 & 0.19 & 0.12 \\
Total drug exposure & 0.01 & 0.02 & 0.13 & 0.56 \\
Weight at last treatment & -10.13 & 11.04 & -0.19 & 0.37 \\
\hline
\end{tabular}

${ }^{\mathrm{a}} R^{2}=0.02, F=0.21 .{ }^{\mathrm{b}} R^{2}=0.05, F=0.43$.

\section{Reproductive output}

Mean reproductive output during the study period did not differ between adult-treated subjects $(M=1.29$, S.D. $=0.99$ births $)$ and age-matched controls $(M=1.57$, S.D. $=0.85$ births; $t(26)=-0.82, P=0.42$, Cohen's $d=0.31$ ). Adolescent-treated subjects had a significantly higher mean reproductive output $(M=2.06$, s.D. $=0.66$ births) than did age-matched $(M=1.29$, s.D. $=0.69$ births; $t(32)=3.32, P=0.002$, Cohen's $d=1.14)$ and parity-matched controls $(M=0.88$, s.D. $=0.49$ births; $t(32)=5.93, P<0.001$, Cohen's $d=2.03)$.

\section{Pregnancy outcomes}

The most commonly observed negative pregnancy outcomes during the study period were neonatal death, maternal abuse/neglect, and stillbirth (Table 4). The incidence of neonatal death did not significantly differ between adult-treated subjects $(17 \%)$ and age-matched controls $(17 \%$; FET, $d f=1, n=36, P=1.00, \Phi=0.00)$ or between adolescent-treated subjects $(12 \%)$ and agematched ( $3 \%$; FET, $d f=1, n=68, P=0.36, \Phi=0.17$ ) or parity-matched controls $(21 \%$; FET, $d f=1, n=68$, $P=0.51, \Phi=0.12$; Table 4).

The incidence of maternal abuse/neglect did not significantly differ between adult-treated subjects $(11 \%)$ and their age-matched controls $(0 \%$; FET, $d f=1, n=36$, $P=0.49, \quad \Phi=0.24)$. However, adolescent-treated subjects had a significantly higher incidence of maternal abuse/neglect $(24 \%)$ than did both their age-matched $(0 \%$; FET, $d f=1, n=68, P=0.01, \Phi=0.37)$ and paritymatched controls $(0 \%$; FET, $d f=1, n=68, P=0.01, \Phi=$ 0.37; Table 4). Looking exclusively at first births within the study period, the incidence of maternal abuse/ neglect did not significantly differ between adolescenttreated subjects $(12 \%)$ and their age-matched $(0 \%$; FET, $d f=1, \quad n=34, P=0.49, \Phi=0.25)$ or parity-matched controls ( $0 \%$; FET, $d f=1, n=34, P=0.49, \Phi=0.25)$.

The incidence of stillbirth did not significantly differ between adult-treated subjects $(11 \%)$ and age-matched controls (6\%; FET, $d f=1, n=36, P=1.00, \Phi=0.10$; Fig. 1). Conversely, adolescent-treated subjects had a significantly higher incidence of stillbirth $(24 \%)$ than did both age-matched ( $0 \%$; FET, $d f=1, n=68, P=0.01, \Phi=$ $0.37)$ and parity-matched controls (3\%; FET, $d f=1$, $n=68, P=0.03, \Phi=0.30$; Table 4; Fig. 1). Looking exclusively at first births within the study period, adolescent-treated subjects still had a significantly higher incidence of stillbirth (29\%) than did both agematched $(0 \%$; FET, $d f=1, n=34, P=0.04, \Phi=0.42)$ and parity-matched controls $(0 \% ; \mathrm{FET}, d f=1, n=34$, $P=0.04, \Phi=0.42$ ).

Looking exclusively within treated subjects, females placed on MPA treatment prior to first ovulation had a significantly higher incidence of stillbirth $(46 \%)$ than did females placed on MPA treatment after first ovulation $(10 \%, \mathrm{FET}, d f=1, n=52, P=0.01, \Phi=0.39$; Fig. 2). When controlling for dam, diabetes, and total drug exposure, MPA exposure prior to first ovulation remained a significant positive predictor of stillbirth after the MPA-treatment cessation (Table 5). When controlling for dam, ovulatory status at MPA-treatment onset, and total drug exposure, diabetes was also a significant positive predictor of stillbirth (Table 5). When controlling for dam, diabetes, and ovulatory status at MPA-treatment onset, total drug exposure was not a significant predictor of stillbirth following MPA-treatment cessation (Table 5). There was no effect of birth number after the final MPA injection (first, second, or third birth) on stillbirth $(F(2,57)=1.00, P=0.37)$. The adolescent-treated subject born to a dam with previous MPA-exposure experienced no negative pregnancy outcomes during the study period.

Table 4 Comparison of pregnancy outcomes during sampling period by treatment and control groups.

\begin{tabular}{|c|c|c|c|c|c|}
\hline Outcome & Adult-treated & Age controls & Adolescent-treated & Age controls & Parity controls \\
\hline Live birth & $10(56)$ & $14(78)$ & $13(38)^{a, b}$ & $33(97)$ & $26(76)$ \\
\hline Planned C-section & 0 & 0 & $1(3)$ & 0 & 0 \\
\hline Stillbirth & $2(11)$ & $1(6)$ & $8(24)^{c, b}$ & 0 & $1(3)$ \\
\hline Neonatal death & $3(17)$ & $3(17)$ & $4(12)$ & $1(3)$ & $7(21)$ \\
\hline Maternal abuse/neglect & $2(11)$ & 0 & $8(24)^{c, b}$ & 0 & 0 \\
\hline Premature birth & $1(6)$ & 0 & 0 & 0 & 0 \\
\hline Total births & 18 & 18 & 34 & 34 & 34 \\
\hline
\end{tabular}

Number of births (\% of total births).

${ }^{a}$ Adolescent-treated significantly differed from age controls at the level of $P<0.001 .{ }^{\mathrm{b}}$ Adolescent-treated significantly differed from parity controls at the level of $P<0.05$. 'Adolescent-treated significantly differed from age controls at the level of $P<0.05$. 


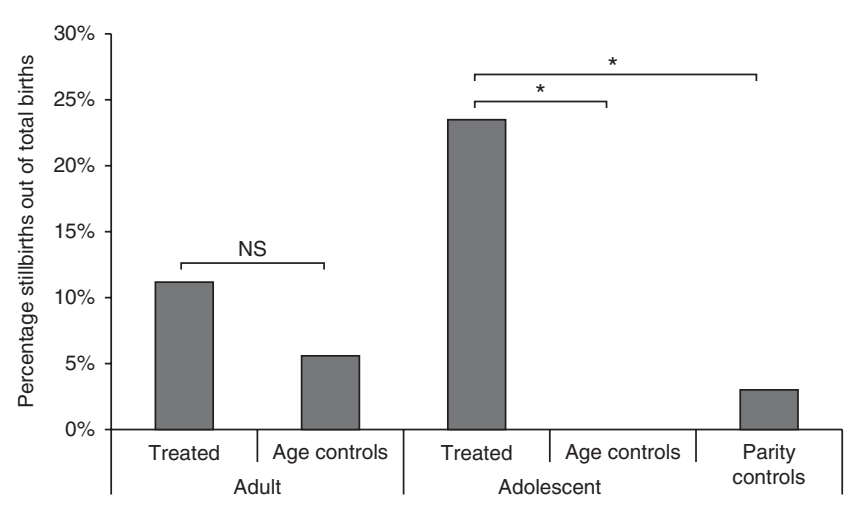

Figure 1 Percentage of total births categorized as stillbirths by treatment group. The incidence of stillbirth was examined over the same number of births for treated subjects and their matched controls. Adult-treated subjects did not differ from their age-matched controls. Adolescenttreated subjects had a significantly higher incidence of stillbirth after MPA-treatment cessation than did their age-matched or parity-matched controls. ${ }^{*} P<0.05 ; \mathrm{NS}$, not significant.

\section{Discussion}

In the present study, all but one MPA-treated female resumed ovulating within the study period following their final MPA injections. There was no difference in latency to return of fertility after the final MPA injection for females placed on MPA treatment as nulliparous adolescents vs parous adults; however; the posttreatment incidence of stillbirth varied significantly with the developmental timing of treatment onset.

\section{Stillbirth}

Females placed on MPA treatment as nulliparous adolescents had an increased incidence of stillbirth post-treatment as compared to both untreated females of the same age and untreated females with equivalent reproductive experience. Conversely, we found no evidence of increased stillbirths post-treatment for females placed on MPA treatment as parous adults, even though the adult-treated females were on treatment longer and were exposed to more MPA over the treatment period than were the adolescent-treated females. The incidence of diabetes did not differ between the adult- and adolescent-treated females, suggesting that diabetes was not the cause of the increased incidence of stillbirth for the adolescent-treated females. When considering only first births, adolescent-treated females still had an increased incidence of stillbirth as compared to their matched controls, indicating that this finding was not related to the fact that some treated females contributed more births toward the total count of births during the study period than did others. Several adolescent-treated females experienced repeated stillbirths, and stillbirths even three pregnancies out from (and more than 3 years after) their final MPA injections, indicating that stillbirth was not the result of lingering MPA (Schwallie \& Assenzo 1974, Fotherby et al. 1980).

Within the treated subjects, we found that females exposed to MPA prior to first ovulation had a significantly higher incidence of stillbirth post-treatment as compared to females placed on MPA treatment at any point after first ovulation (as nulliparous ovulatory adolescents or parous adults). In total, females placed on MPA treatment prior to first ovulation had a stillbirth rate of $46 \%$, as compared to only $10 \%$ for females placed on MPA treatment after first ovulation (Fig. 2). We also found that, within treated subjects, diabetic females had an increased incidence of stillbirth as compared to nondiabetic females. This finding is consistent with findings from studies of pregnancy outcomes in diabetic women, in which diabetes has been shown to be a risk factor for stillbirth (Lauenborg et al. 2003, Wood et al. 2003, Mathiesen et al. 2011). Importantly, even when controlling for diabetes, exposure to MPA prior to first ovulation was still a positive predictor of stillbirth. These findings indicate that diabetes and exposure to MPA prior to first ovulation are independent risk factors for stillbirth.

Taken together, these findings strongly suggest that MPA exposure increased the likelihood of stillbirth posttreatment only for females placed on MPA-treatment in early adolescence and that MPA exposure most severely affected females placed on MPA-treatment prior to first ovulation. This novel finding raises the question of whether there is a developmental window of vulnerability during which exposure to chronically elevated

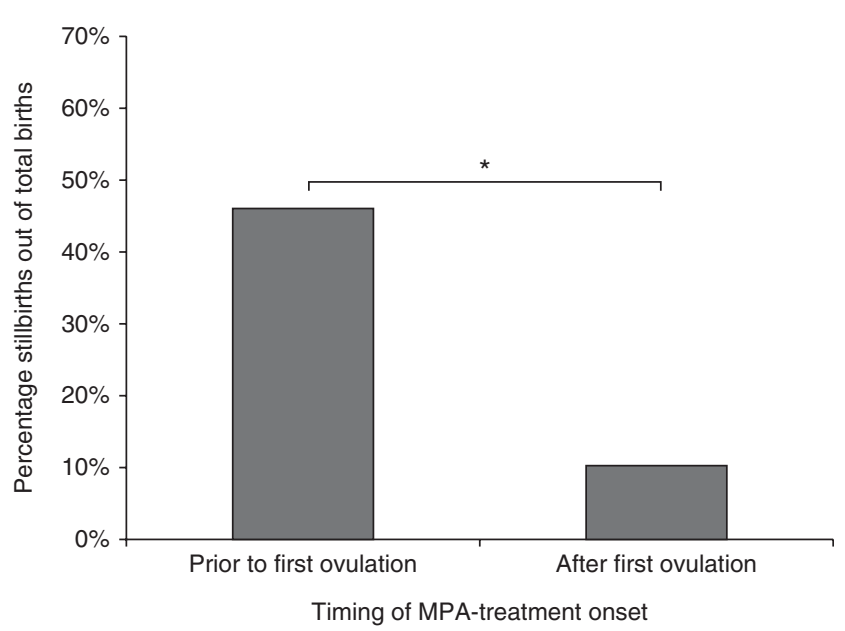

Figure 2 Percentage of total births categorized as stillbirths by ovulatory status at MPA-treatment onset. Females placed on MPA treatment prior to first ovulation had a significantly higher incidence of stillbirth after MPA-treatment cessation than did females placed on MPA treatment after first ovulation. When controlling for dam, diabetes, and total drug exposure, ovulatory status at treatment onset was still a significant predictor of stillbirth, with exposure to MPA prior to first ovulation being positively associated with stillbirth (Table 5). ${ }^{*} P<0.05$. 
Table 5 Summary of nested binary logistic regression model predicting stillbirth.

\begin{tabular}{lrrrc}
\hline Predictors & $\boldsymbol{B}$ & s.E.M. & Wald & $\boldsymbol{P}$ \\
\hline Constant & 6.11 & 3.10 & 3.90 & 0.05 \\
Diabetes & 2.85 & 1.35 & 4.48 & $0.03^{*}$ \\
Ovulatory status at first treatment & -3.81 & 1.05 & 13.29 & $<0.01^{*}$ \\
Total drug exposure & $<0.01$ & $<0.01$ & 3.34 & 0.07 \\
\hline
\end{tabular}

The dependent variable in this analysis is stillbirth coded so that $0=$ no and $1=$ yes. Ovulatory status at first treatment is coded so that $0=$ preovulatory and $1=$ ovulatory. Diabetes is coded so that $0=$ no diabetes and $1=$ diabetes. *Significant at the level of $P<0.05$.

levels of progestin permanently and negatively alter future reproductive capacity.

\section{Maternal abuse/neglect}

The adolescent-treated females were more likely to abuse and/or neglect their infants than were their age-matched and parity-matched controls. Although we do not know the cause, this high incidence of aberrant maternal behavior in the adolescent-treated females may reflect a lack of pre-adult experience with infants. All adult females living in the social group containing the adolescent-treated females were placed on MPA treatment while the adolescent-treated females were still juveniles; consequently, these females had no opportunity to interact with infants until they gave birth to their own.

\section{Return of fertility and reproductive output}

The treated females showed considerable individual variation in latency to resumption of ovulation after their final MPA injections. Consistent with findings from studies of human MPA users (Pardthaisong et al. 1980, Lan et al. 1984, Garzes-Flores et al. 1985), latency to resumption of ovulation was seemingly unrelated to reproductive experience prior to treatment, weight at last treatment, or total drug exposure. Women show considerable variation in the rate at which they metabolize MPA (Schwallie \& Assenzo 1974, Fotherby et al. 1980), and individual differences in MPA metabolism and clearance were likely responsible for the observed variation in latency to resumption of ovulation after the final MPA injection.

We found no evidence that MPA exposure negatively impacted post-treatment rates of conception for either adult- or adolescent-treated females. On average, the adult-treated females gave birth as often as did their matched controls during the study period, and the adolescent-treated females actually gave birth more often than did their matched controls. These results are consistent with findings from studies of human MPA users, in which rates of conception have been shown not to differ between former MPA users and age-matched controls (Schwallie \& Assenzo 1974). The increased reproductive output of the adolescent-treated females was likely related to their increased incidence of infant mortality. Female primates experience lactational amenorrhea, which lengthens the interbirth interval (Schallenberger et al. 1981, Stewart 1988, Recabarren et al. 2000). The death of an infant removes lactational amenorrhea, and mothers who lose an infant typically conceive again more quickly than do mothers who successfully wean an infant; therefore, more frequent infant loss increases reproductive output but not reproductive success. When interpreting the effects of MPA exposure on future fertility, it is crucial to distinguish between the ability to conceive and the ability to successfully reproduce. The current literature emphasizes post-treatment conception rates for human MPA users but neglects to investigate pregnancy outcomes following conception.

\section{Speculations}

We can only speculate as to why exposure to MPA during early adolescence might increase the risk of stillbirth in adulthood in primates. One possibility is that adolescent MPA exposure alters the structure of the adult uterus. In primates, the uterus continues to develop into puberty, and this postnatal uterine development is sensitive to progestins (Yin \& Ma 2005). Evidence suggests that exposure to elevated levels of progestins during species-specific sensitive periods of uterine development can permanently alter adult uterine structure (for review, see Gray et al. 2001). In the current study, it is possible that exposure to MPA during early adolescence, a critical period of uterine development, permanently altered the structure of the uterus and increased the likelihood of stillbirth in adulthood in the adolescent-treated females.

A second possibility is that adolescent MPA exposure disrupts the oxytocin system. Oxytocin has been shown to be critical for the upregulation of uterine contraction strength during labor and the completion of parturition and for the expression of species-typical maternal behavior in a variety of mammalian species (Keverne \& Kendrick 1992, Pedersen et al. 1992, Olazabal \& Young, 2006). Therefore, a disruption of the oxytocin system could account for both the increased incidence of stillbirth and the increased incidence of aberrant maternal behavior in the adolescent-treated females. Progestins suppress uterine contractility during pregnancy, in part, by binding and blocking the uterine oxytocin receptor (OTR; Grazzini et al. 1998). Because progestins show an affinity for OTR, exposure to MPA during early adolescence could potentially disrupt the oxytocin system. Whether MPA exposure during adolescence is associated with decreased levels of circulating oxytocin during labor and/or following parturition, or decreased expression of OTR in the brain and/or uterus in adulthood, remains to be explored. 


\section{Conclusions}

The findings of the present study point to a relationship between MPA exposure during early adolescence and an increased likelihood of stillbirth in adulthood, years after MPA treatment has ended. These findings suggest that MPA exposure during early adolescence may permanently decrease post-treatment reproductive capacity, particularly for females placed on MPA treatment prior to first ovulation. The Depo-Provera product insert states that Depo-Provera should not be used prior to menarche; however, the insert fails to address the use of DepoProvera after menarche but prior to first ovulation (Pfizer Canada Inc. 2013). Given that female sooty mangabeys share many aspects of reproductive physiology and endocrinology with women, the results of the present study raise questions about the safety of administering MPA to adolescent girls.

\section{Declaration of interest}

The authors declare that there is no conflict of interest that could be perceived as prejudicing the impartiality of the research reported.

\section{Funding}

This clinical records study was conducted at the Yerkes National Primate Research Center Field Station, an AAALAC accredited animal research facility. This study was funded by the National Center for Research Resources P51RR165 and is currently supported by the Office of Research Infrastructure Programs/OD P51OD11132. This study was further supported by the National Science Foundation Graduate Training Fellowship. A subset of the findings reported here were presented in an oral presentation at the Society for the Study of Reproduction Annual Meeting in Montreal, Quebec, Canada (July 2013).

\section{Acknowledgements}

The authors thank Dr Maria Crane and Dr Joyce Cohen for their helpful feedback on an earlier draft of this paper.

\section{References}

Aidara D, Badawi M, Tahiri-Zagret C \& Robyn C 1981 Changes in concentrations of serum prolactin, $\mathrm{FSH}$, oestradiol and progesterone and of the sex skin during the menstrual cycle in the mangabey monkey (Cercocebus atys lunulatus). Journal of Reproduction and Fertility 62 475-481. (doi:10.1530/jrf.0.0620475)

Bender R \& Lange S 2001 Adjusting for multiple testing - when and how? Journal of Clinical Epidemiology 54 343-349. (doi:10.1016/S08954356(00)00314-0)

Cohen J. 1977 Statistical Power Analysis for the Behavioral Sciences. New York, NY: Academic Press, Inc.

Cromer BA, Berg-Kelly KS, van Groningen JP, Seimer BS \& Ruusuvaara L 1998 Depot medroxyprogesterone acetate (Depo-Provera) and levonorgestrel (Norplant) use in adolescents among clinicians in Northern Europe and the United States. Journal of Adolescent Health 23 74-80. (doi:10.1016/S1054-139X(98)00030-5)
De Vleeschouwer K, Leus K \& Van Elsacker L 2000 An evaluation of the suitability of contraceptive methods in golden-headed lion tamarins (Leontopithecus chrysomelas), with emphais on melengestrol acetate (MGA) implants: (I) Effectiveness, reversibility and medical side-effects. Animal Welfare 9 251-271.

Fotherby K, Koetsawang S \& Mathrubutham M 1980 Pharmacokinetic study of different doses of Depo Provera. Contraception 22 527-536. (doi:10.1016/0010-7824(80)90105-5)

Garzes-Flores J, Cardenas S, Rodriguez V, Cravioto MC, Diaz-Sanchez V \& Perez-Palacios G 1985 Return of ovulation following the use of longacting injectable contraceptives: a comparative study. Contraception 31 361-366. (doi:10.1016/0010-7824(85)90004-6)

Gray CA, Bartol FF, Tarleton BJ, Wiley AA, Johnson GA, Bazer FW \& Spencer TE 2001 Developmental biology of uterine glands. Biology of Reproduction 65 1311-1323. (doi:10.1095/biolreprod65.5.1311)

Grazzini E, Guillon G, Mouillac B \& Zingg HH 1998 Inhibition of oxytocin receptor function by direct binding of progesterone. Nature 392 509-512. (doi:10.1038/33176)

Greenwood R \& McCaw-Binns A 1994 Does maternal behaviour influence the risk of perinatal death in Jamaica? Paediatric and Perinatal Epidemiology 8 (s1) 54-65. (doi:10.1111/j.1365-3016.1994. tb00491.x)

Greenwood R, Foster-Williams K, Ashley D, Keeling J \& Golding J 1994 The epidemiology of antepartum fetal death in Jamaica. Paediatric and Perinatal Epidemiology 8 (s1) 98-109. (doi:10.1111/j.1365-3016.1994. tb00494.x)

Hadidian J \& Bernstein IS 1979 Female reproductive cycles and birth data from an Old World monkey colony. Primates 20 429-442. (doi:10.1007/ BF02373394)

Jones AC, Herndon JG, Courtney CL, Collura L \& Cohen JK 2014 Clinicopathologic characteristics, prevalence, and risk factors of spontaneous diabetes in sooty mangabeys (Cercocebus atys). Comparative Medicine 64 200-210.

Keverne EB \& Kendrick KM 1992 Oxytocin facilitation of maternal behavior in sheep. Annals of New York Academy of Sciences 652 83-101. (doi:10.1111/j.1749-6632.1992.tb34348.x)

Khoiny FE 1996 Use of Depo-Provera in teens. Journal of Pediatric Health Care 10 195-201. (doi:10.1016/S0891-5245(96)90001-2)

Lan PT, Aedo AR, Landgren BM, Johannisson E \& Diczfalusy E 1984 Return of ovulation following a single injection of depo-medroxyprogesterone acetate: a pharmacokinetic and pharmacodynamic study. Contraception 29 1-18. (doi:10.1016/0010-7824(84)90054-4)

Lauenborg J, Mathiesen E, Ovesen P, Westergaard JG, Ekbom P, MølstedPedersen L \& Damm P 2003 Audit on stillbirths in women with pregestational type 1 diabetes. Diabetes Care 26 1385-1389. (doi:10.2337/ diacare.26.5.1385)

Mathiesen ER, Ringholm L \& Damm P 2011 Stillbirth in diabetic pregnancies. Best Practice \& Research. Clinical Obstetrics \& Gynaecology 25 105-111. (doi:10.1016/j.bpobgyn.2010.11.001)

Mosher WD \& Jones J 2010 Use of contraception in the United States: 1982-2008. Vital and Health Statistics 29 1-44. Series 23, Data from the National Survey of Family Growth.

Olazabal DE \& Young LJ 2006 Oxytocin receptors in the nucleus accumbens facilitate "spontaneous" maternal behavior in adult female prairie voles. Neuroscience 141 559-568. (doi:10.1016/j.neuroscience. 2006.04.017)

Pardthaisong T, Gray RH \& McDaniel EB 1980 Return of fertility after discontinuation of depot medroxyprogesterone acetate and intra-uterine devices in northern Thailand. Lancet 8 509-511. (doi:10.1016/S01406736(80)92765-8)

Pedersen CA, Caldwell JD, Peterson G, Walker CH \& Mason GA 1992 Oxytocin activation of maternal behavior in the rata. Annals of New York Academy of Sciences 652 58-69. (doi:10.1111/j.1749-6632.1992. tb34346.x)

Pfizer Canada Inc. 2013 DEPO-PROVERA/DEPO-PROVERA-SC (medroxyprogesterone acetate)-Product Monograph. Retrieved from: http://www. pfizer.ca/en/our_products/products/monograph/181

Recabarren MP, Vergara M, Martınez MC, Gordon K \& Serón-Ferré M 2000 Impact of lactation upon fertility in the New World primate capuchin monkey (Cebus apella). Journal of Medical Primatology 29 350-360. (doi:10.1034/j.1600-0684.2000.290507.x) 
Rivera R, Yacobson I \& Grimes D 1999 The mechanism of action of hormonal contraceptives and intrauterine contraceptive devices. American Journal of Obstetrics and Gynaecology 181 1263-1269. (doi:10.1016/S0002-9378(99)70120-1)

Schallenberger E, Richardson DW \& Knobil E 1981 Role of prolactin in the lactational amenorrhea of the rhesus monkey (Macaca mulatta). Biology of Reproduction 25 370-374. (doi:10.1095/biolreprod25.2.370)

Schwallie PC \& Assenzo JR 1974 The effect of depo-medroxyprogesterone acetate on pituitary and ovarian function, and the return of fertility following its discontinuation: a review. Contraception 10 181-202. (doi:10.1016/0010-7824(74)90073-0)

Stewart KJ 1988 Suckling and lactational anoestrus in wild gorillas (Gorilla gorilla). Journal of Reproduction and Fertility 83 627-634. (doi:10.1530/ jrf.0.0830627)

Tolaymat LL \& Kaunitz AM 2007 Long-acting contraceptives in adolescents. Current Opinion in Obstetrics and Gynecology 19 453-460. (doi:10.1097/GCO.0b013e3282ef1cd2)
Wood C, Ballou JD \& Houle CS 2001 Restoration of reproductive potential following expiration or removal of melengestrol acetate contraceptive implants in golden lion tamarins (Leontopithecus rasalia). Journal of Zoo and Wildlife Medicine 32 417-425. (doi:10.1638/1042-7260 (2001)032 [0417:RORPFE]2.0.CO;2)

Wood SL, Jick H \& Sauve R 2003 The risk of stillbirth in pregnancies before and after the onset of diabetes. Diabetic Medicine 20 703-707. (doi:10.1046/j.1464-5491.2003.01015.x)

Yin Y \& Ma L 2005 Development of the mammalian female reproductive tract. Journal of Biochemistry 137 677-683. (doi:10.1093/jb/mvi087)

Received 8 July 2015

First decision 24 August 2015

Revised manuscript received 18 September 2015

Accepted 30 September 2015 